\title{
Superposition rheology and anisotropy in rheological properties of sheared colloidal gels
}

\author{
Gabriele Colombo \\ Department of Materials, ETH Zürich, CH-8093 Zürich, Switzerland \\ Sunhyung Kim \\ Department of Chemical Engineering, KU Leuven, University of Leuven, Celestijnenlaan 200F, \\ B-3001 Leuven, Belgium \\ Thomas Schweizer \\ Department of Materials, ETH Zürich, CH-8093 Zürich, Switzerland \\ Bram Schroyen, Christian Clasen, and Jan Mewis \\ Department of Chemical Engineering, KU Leuven, University of Leuven, Celestijnenlaan 200F, \\ B-3001 Leuven, Belgium \\ Jan Vermant ${ }^{\text {a) }}$ \\ Department of Materials, ETH Zürich, CH-8093 Zürich, Switzerland
}

(Received 13 March 2017; final revision received 25 July 2017; published 17 August 2017)

\begin{abstract}
Gelling colloidal suspensions represent an important class of soft materials. Their mechanical response is characterized by a solid-to-liquid transition at a given shear stress level. Moreover, they often exhibit a complex time-dependent rheological behavior known as thixotropy. The viscosity changes find their origin in the microstructure, which depends on flow history. Yet, the structural response of colloidal gels to flow differs fundamentally from most complex fluids, where flow induces orientation. Upon yielding, low to intermediate volume fraction gels break down in a spatially anisotropic way. Bonds in the velocity-velocity gradient plane are broken, whereas microstructural features in other planes are less affected. The subsequent flow-induced microstructural anisotropy is characterized by typical butterfly scattering patterns. However, as yet there was no evidence for the pertinence of this anisotropy for the rheological properties of these systems. In the present work, orthogonal superposition rheometry was first used to evaluate how the flow-induced microstructure affects the viscoelastic properties. It was shown to retain significant elasticity in the velocity-vorticity plane, even when the structure liquefied. Further, the shearinduced mechanical anisotropy was measured using two-dimensional small amplitude oscillatory shear, exploiting the fact that for suitable thixotropic samples the recovery after arresting the flow is relatively slow. It was hence possible to measure the anisotropy of the moduli upon cessation of flow. The mechanical anisotropy was shown to be spectacular, with the storage moduli in perpendicular directions differing by as much as 2 orders of magnitude. (C) 2017 The Society of Rheology. [http://dx.doi.org/10.1122/1.4998176]
\end{abstract}

\section{INTRODUCTION}

Gelling colloidal suspensions have a wide range of advanced technological and biomedical applications. Recent examples include 3D-direct printing [1], tissue scaffolding [2], or even molecular gastronomy [3]. The wide range of utilization of these materials is the result of the solidlike behavior at rest, combined with their ability to liquefy when subjected to mechanical stress. In terms of material functions, the rheological properties of flocculated suspensions

\footnotetext{
a) Author to whom correspondence should be addressed; electronic mail: jan.vermant@mat.ethz.ch
}

are described by an elasticity that depends on shear history, a yield stress, and a viscosity that not only changes reversibly with shear rate but also with time. The latter effect is known as thixotropy [4].

These rheological effects find their origin in the microstructure and its response to flow. The calculation of the linear viscoelastic properties of aggregated suspensions requires taking into account the effect of the microstructure on both hydrodynamic and thermodynamic stresses. The high frequency response is expected to be similar to that of hard sphere suspensions [5,6]. At low frequencies, the oscillatory deformation is opposed by the gel microstructure and several contributions can lead to a broad relaxation spectrum. Various approaches have been proposed to explain 
these properties, with most theories focusing on the structure at rest. For strong gels, fractal scalings $[7,8]$ and poroelastic models [9] have been proposed linking the elasticity of the gel network to the rigidity of intra- or interfloc links $[7,10]$. In weaker gels with more of a bicontinuous, disordered morphology, it has been suggested that the elasticity is determined by the localization of particles in the gel, which slows down the Brownian relaxation processes. Mode coupling theory has then been used to describe the linear viscoelastic spectra under quiescent conditions $[11,12]$.

In addition to physicochemical details (e.g., the volume fraction, the pair potential), the flow history plays an important role in the details of the microstructure, such as the local coordination number and the fractal dimensions, as well as in the resulting mechanical properties [4]. During flow, the network structure will break down, and the stresses acting on the individual aggregates will, in turn, alter their internal structure. However, it is not a priori clear how stresses are transmitted once the material loses connectivity and the structure reorganizes. Most existing models assume an isotropic gel structure. For the fractal gels, the non-Newtonian response is often described by a shear rate dependence of an isotropic floc size and density [13], resulting from the hydrodynamic forces that act on these aggregates. In the framework of mode coupling theory, nonlinear rheological effects are ascribed to changes in the localization length scale due to deformation of the microstructure. However, recently Hsiao et al. [14] argued that localization may not be a good descriptor of gels upon yielding, and suggested that the main contribution to the elasticity derives from a subpopulation of slow, isostatic clusters, which have long relaxation times [15]. Significant viscoelasticity was experimentally observed during flow in depletion gels consisting of fluorescent poly(methyl methacrylate) particles. Their structure was locked-in by photopolymerization after step-strain nonlinear deformations and subsequently evaluated by confocal microscopy [14]. The structure-property analysis suggested that the elasticity could be attributed to the slow Brownian relaxation of rigid, isostatic clusters. Recently, "ideal thixotropy" was defined as a purely viscous time dependent response to the history of the strain rate [16], in an effort to clearly distinguish thixotropy from ordinary nonlinear elasticity. The presence of significant residual elasticity in sheared colloidal gels mentioned earlier, however, indicates that such a purely viscous response is only the limiting case of real behavior. This argument is reinforced by the present work, where a colloidal gel at low volume fraction is investigated, and elasticity under flow is observed. Indeed, if at all, ideal thixotropy is expected to be found in dilute systems. Clearly, predicting the rheological properties of these systems remains challenging. Theoretical approaches fundamentally differ and do not adequately predict all rheological properties or flow history effects [4], thus calling for further work.

In order to study the nonlinear rheological properties of colloidal gels, different experimental approaches can be used. An increasingly popular technique is large amplitude oscillatory shear (LAOS), which has also been applied to flocculated thixotropic dispersions [17]. However, while the frequency dependency offers the possibility to probe different time and length scales, this method relies on subjecting the sample to a very complex kinematic history, which is an issue especially for thixotropic gels with a microstructure depending in a complex manner on the previously experienced shear history. Another approach, which still enables frequency dependent measurements while retaining clean kinematics, consists in superimposing a small oscillation on a steady shear flow. Similar to linear viscoelasticity, the response to the superimposed oscillatory motion can be analyzed in terms of storage and loss superposition moduli. Parallel superposition is the easier experiment to perform, but the physical meaning of the parallel moduli is not straightforward, so that parallel superposition moduli can even be negative. In the case of superposition in the direction perpendicular to flow the moduli are more closely related to normal linear viscoelastic behavior [18] (see Sec. II). Therefore, this orthogonal superposition rheometry (OSR) is very well suited to study the flow behavior of colloidal gels, allowing to probe how the different relaxation times of the material are affected by shear, and to critically assess the role of residual aggregates upon flow and structure breakdown, as well as the presence of residual elasticity. The moduli under steady state flow conditions are still expected to reflect the effects of a shear rate dependent and anisotropic suspension microstructure. This approach is further motivated by the results on nonaqueous layered silicate suspensions [19], where mechanical anisotropy in a polymer-clay nanocomposite was shown for the first time, using 2D-small amplitude oscillatory shear (2D-SAOS). Such systems are, however, made up of platelike particles, making them less ideal to interrogate flocculated suspension mechanics in general. Moreover, we aim to study the moduli during flow to interrogate the mechanical consequences of the changing microstructure in sheared aggregated suspensions.

The response of the suspension microstructure to deformation or flow has been studied for a variety of flocculated systems with spherical building blocks [20]. For individual aggregates, whether they break-up, erode or get stretched in the flow direction has been well established, depending on the flow conditions (see, e.g., [21] and references therein). For percolated colloidal gels, the situation is more complicated. Weakly aggregated or reversible, physical gel networks are intrinsically inhomogeneous, and the deformation can be expected to be localized in the weaker regions of the network, whereas stronger regions are densified. The vorticity inherent to shear flow causes this process to be subtle. Continuous break-up and subsequent reaggregation lead to a local compaction along the compressional axis of the flow field with an increasingly heterogeneous structure [22,23]. Under an imposed low shear rate, the microstructure of suspensions that form a percolating network at rest can, hence, be expected to be anisotropic in nature upon deformation. This anisotropy has been amply evidenced by a wide array of scattering measurements, which typically reveal a pronounced butterfly shaped scattering pattern (small angle light (SALS), neutron (SANS) and X-ray (SAXS) scattering) [17,24-26] and by some recent direct microscopic observations in both $2 \mathrm{D}$ $[21,22,27]$ and 3D [23,28]. The nonequilibrium structure factor has a complex spatial dependence with a local anisotropy 
in the velocity gradient, which develops along the compressional axis of flow $[17,26]$. Using flow SALS, (Ultra)SAXS or SANS a butterfly pattern is observed in the velocityvorticity plane of flow [17,24-26] accompanied by a similar anisotropy in the velocity-velocity gradient plane [17,26]. Detailed flow-SANS measurements show how these anisotropic structure factors (on length scales corresponding to a few particle diameters) correlate with rheology [17,29]. USAXS and SALS measurements show how these anisotropic microstructures extend to large length scales, beyond hundreds of particle diameters [26], which is consistent with the recent microscopic investigations.

Differing from materials where flow induces microstructural changes by orientation and stretching [30-33] in percolated reversible colloidal gels, the anisotropic structure factors are a consequence of a spatially selective breakdown of the colloidal network. The butterfly scattering pattern is due to the systematic loss of structure in the flow direction, and even an initially isotropic structure factor gets constricted in the direction of flow. In the velocity-vorticity plane, the largest structures are initially unchanged as no significant forces act in the neutral direction $[17,20,25,26]$. For confined suspensions even log-rolling structures (rouleau formation) have been observed [34], but it is as yet unclear as to whether they also occur in bulk flow and are related to the observed butterfly scattering patterns. The anisotropy and other characteristic aspects of the microstructure evolve over time scales much beyond the viscoelastic relaxation time scale of particles or aggregates. Most microstructural evidence concerning the anisotropy of colloidal networks has been to a large extent disregarded in current rheological modeling $[7,13,35,36]$ because implications and measurements of this anisotropy for rheology have only rarely been addressed. As yet there was no evidence of mechanical anisotropy in these systems.

Therefore, in the present work, the relevance of the structural anisotropy on the mechanical properties is investigated. On the one hand, we use OSR to elucidate the mechanical properties of the structure in the velocity-vorticity plane of flow and its changes in microstructure upon breakdown and liquefaction. Second, we quantify the mechanical anisotropy by measuring the moduli in different straining directions, following a preshear step. We use a well characterized model system for investigating gelling thixotropic suspensions, introduced earlier by Dullaert and Mewis [37]. It shows all of the hallmark features of colloidal gel mechanics, including yield stress, shear thinning, and thixotropy. As these systems have a relatively slow recovery, the flow-induced mechanical anisotropy can be quantified by measuring the moduli in two directions after arresting the flow, thus avoiding the problems associated with parallel superposition experiments [18]. This particular system also shows no measurable wall slip [37] and the moduli lie well within the experimental measurement window of the orthogonal superposition device.

\section{MATERIALS AND METHODS}

The model system studied here was formulated by Dullaert and Mewis [37] for studies of thixotropy. The latter authors reported the details concerning the roles of its different components, as well as the specific conditions required to obtain reproducible results and ensure homogeneous flow in the absence of wall slip. Fumed silica particles (Aerosil R972), kindly provided by Grolman, Basel, were dispersed in a highly refined paraffin oil (Sigma-Aldrich, 18512). A low molecular weight poly(isobutene) (PIB: Oppanol B3, BASF) was added to increase the medium viscosity to $0.4 \mathrm{~Pa}$ $\mathrm{s}$ at $25^{\circ} \mathrm{C}$ and to reduce wall slip [37]. The particles were added in five steps to the paraffin oil. A fraction (15\%) of the total, final mass of PIB was added in the first step to take advantage of its adsorption on the silica particles [37]. Between steps, the sample was mixed with an Ultra-Turrax device (IKA T25 digital, S25N-10G disperser) at $8000 \mathrm{rpm}$ and tip sonicated under stirring (Hielscher UP400S). The remaining PIB was then added (totaling $27 \% \mathrm{w} / \mathrm{w}$ ), reaching a final volume fraction of silica $\phi=2.9 \%$.

The flow curve of the final dispersion is shown in Fig. 1, and exhibits the typical features of a flocculated suspension, with a dynamic yield stress of 4.2 Pa. The steady state flow curve was found to be independent of measurement geometry, confirming the absence of slip and inhomogeneities or flow instabilities in the sample. The degree of dispersion is an important factor affecting the rheological properties of this model system, which is, thus, susceptible to aging effects. The measurement protocol included a preshear step at $10 \mathrm{~s}^{-1}$ for $300 \mathrm{~s}$, necessary to ensure reproducible initial conditions by eliminating prior shear history effects. It was verified that the same shear stress was always obtained for this somewhat arbitrary initial state. Subsequently, the sample was sheared at the desired shear rate for a time sufficient to reach steady state, before starting measurements. One sample was allowed to rest for $5400 \mathrm{~s}$ immediately following the preshear step, resulting in a reference "rest" condition.

The double wall Couette flow cell used in the present work is shown in Fig. 2. It is designed to minimize the backflow of material in the measurement gaps $(0.5 \mathrm{~mm})$, due to the pumping effect caused by the oscillatory motion of the bob in the $z$ direction during orthogonal superposition

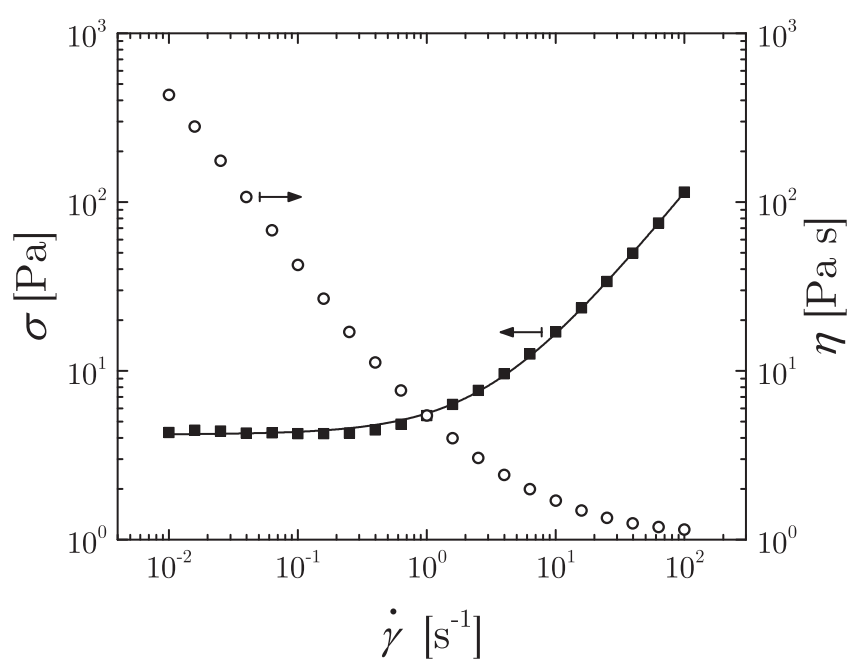

FIG. 1. Steady state flow curve of the studied colloidal dispersion: Shear stress $\sigma$ and viscosity $\eta$ as a function of shear rate $\dot{\gamma}$. The line shows a fit to the Herschel-Bulkley model, giving a dynamic yield stress $\sigma_{y}=4.2 \mathrm{~Pa}$. 


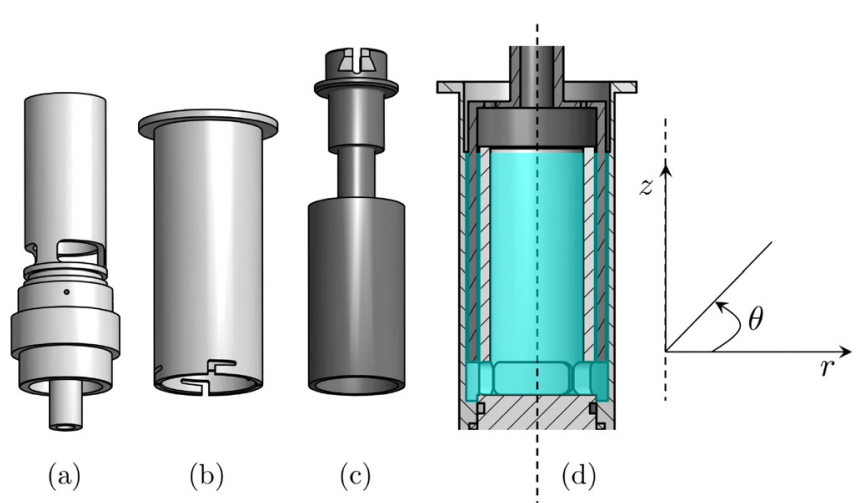

FIG. 2. Double wall Couette cell for OSR. The inner (a) and outer (b) part of the cup, as well as the measuring bob (c) are shown from left to right. The cross section of the assembled cell in measuring position is shown on the right (d), together with the corresponding cylindrical coordinate system. The sample filling is shown in blue/light grey with the large reservoir in the center.

measurements [38]. To reduce the backflow in the gap, it features openings in the inner wall of the cup, allowing the sample to flow in and out of the large central reservoir during measurements. However, for the fluid used here, which is building up a yield stress, this reduction of the pumping flow is less effective than for fluids with a Newtonian low-shear viscosity, as the unsheared fluid in the reservoir can become more difficult to displace. For estimating the effects of the pumping flow, computational fluid dynamics (CFD) calculations (see the Appendix) were performed for the two limiting cases of an open bottom (with flow into the reservoir) and closed bottom of the measuring cell. For the sake of simplicity, these calculations were carried out for a Newtonian fluid. Shear thinning effects will weaken the effects seen for the closed bottom case so that the results are to be understood as upper and lower bounds. In the case of an open bottom, the experimentally measured moduli in the orthogonal direction are overestimated by $10 \%$, mainly due to a pressure contribution on the bottom of the bob. For the case of closed cup wall, both the pressure contribution and the enhanced flow in the measurement gap would cause an increase of the apparent moduli of 2 orders of magnitude. A full calculation for non-Newtonian and thixotropic materials lies beyond the scope of the current work but would be worthwhile to pursue.

The kinematics for the superposition of an oscillatory motion on the steady shear flow are given in Eq. (1), according to the coordinate system of Fig. 2. In the present work, the direction in which measurements are performed are always referred to with respect to the standard angular motion of the rheometer, that is, with respect to the main steady shear flow. Therefore, $\theta$ is the velocity direction, $r$ the velocity gradient direction, and $z$ the vorticity direction

$$
\begin{aligned}
& \theta(t)=\theta\left(t^{\prime}\right)+\left[\dot{\gamma}\left(t-t^{\prime}\right)+\alpha \gamma_{0}\left(\sin \omega t-\sin \omega t^{\prime}\right)\right] r(t), \\
& r(t)=r\left(t^{\prime}\right), \\
& z(t)=z\left(t^{\prime}\right)+\beta \gamma_{0}\left(\sin \omega t-\sin \omega t^{\prime}\right) r(t) .
\end{aligned}
$$

$t$ and $t^{\prime}$ are the present and reference time, respectively, $\omega$ is the frequency of oscillation and $\dot{\gamma}=\left(\partial v_{\theta} / \partial r\right)-\left(v_{\theta} / r\right)$ the shear rate in cylindrical coordinates, with $v_{\theta}$ the velocity in the flow direction. The small amplitude oscillatory flow can be superposed in either parallel $(\alpha=1, \beta=0)$ or orthogonal $(\alpha=0, \beta=1)$ direction with respect to the steady shear flow in $\theta$. Most commercial rheometers are able to perform parallel superposition measurements, which do not require modifications to the hardware. To demonstrate the complexity of the parallel superposition experiment, Yamamoto associated a perturbation spectrum $H\left(\tau, I I_{2 \mathrm{D}}\right)$ with each shear rate, $\tau$ being the relaxation time [39]. The parallel oscillatory flow is strongly coupled with the steady shear [see Eq. (1)], which is reflected in the second invariant of the rate of deformation tensor $2 \mathbf{D}$

$$
I_{2 \mathbf{D}}=\dot{\gamma}^{2}+2 \alpha \gamma_{0} \omega \dot{\gamma} \cos \omega t^{\prime}+(\alpha+\beta) \mathcal{O}\left(\gamma_{0}^{2}\right)
$$

In superposition flows with small amplitudes, i.e., for small perturbations of $I I_{2 \mathrm{D}}$ around $\dot{\gamma}^{2}$, the perturbation spectrum $H\left(\tau, I I_{2 \mathbf{D}}\right)$, can be linearized around the steady state value

$$
\begin{aligned}
H\left(\tau, I I_{2 \mathbf{D}}\right)= & H\left(\tau, \dot{\gamma}^{2}\right)+2 \alpha \gamma_{0} \omega \dot{\gamma} \cos \omega t^{\prime} \frac{\partial H\left(\tau, \dot{\gamma}^{2}\right)}{\partial\left(\dot{\gamma}^{2}\right)} \\
& +(\alpha+\beta) \mathcal{O}\left(\gamma_{0}{ }^{2}\right) .
\end{aligned}
$$

For parallel superposition $(\alpha=1, \beta=0)$, the derivative in the cross term of the linearized spectrum complicates the interpretation of the spectra and the corresponding moduli.

The orthogonal superposition $(\alpha=0, \beta=1)$ case yields directly rate-dependent spectra and superposition moduli that retain the same physical meaning of storage and loss modulus as those of linear viscoelasticity when the amplitude is small [18]. Flow induced anisotropy may make the spectral nature of the response anisotropic, which is not captured by the simple Yamamoto analysis. Superposition moduli can help designing or evaluating constitutive models, by assessing the effects of flow on the spectra in a direct and elegant manner [40,41].

A strain-controlled rheometer (ARES-G2, TA Instruments) is used to perform orthogonal superposition measurements. For the latter, the control loop of the normal force transducer is adapted to generate an axial motion, orthogonal to the standard angular motion of the rheometer [38,41]. The maximum deformation in the axial direction is limited $(50 \mu \mathrm{m})$, but suffices for linear oscillatory measurements. In this manner, an oscillation with variable frequency $\omega$ can be superimposed onto a steady flow at $\dot{\gamma}$ in an orthogonal superposition experiment. The oscillatory measurement can be continued after the main flow has been arrested, even in different straining directions, separately or combined (2D-SAOS) $[19,42]$, as will be discussed further.

\section{RESULTS AND DISCUSSION}

The orthogonal superposition experiments allow the identification of the transition from flow at the yield stress plateau (plastic flow) to the viscous flow regime more clearly. The orthogonal superposition moduli, i.e., those observed during steady state flow conditions, are shown in Fig. 3, 

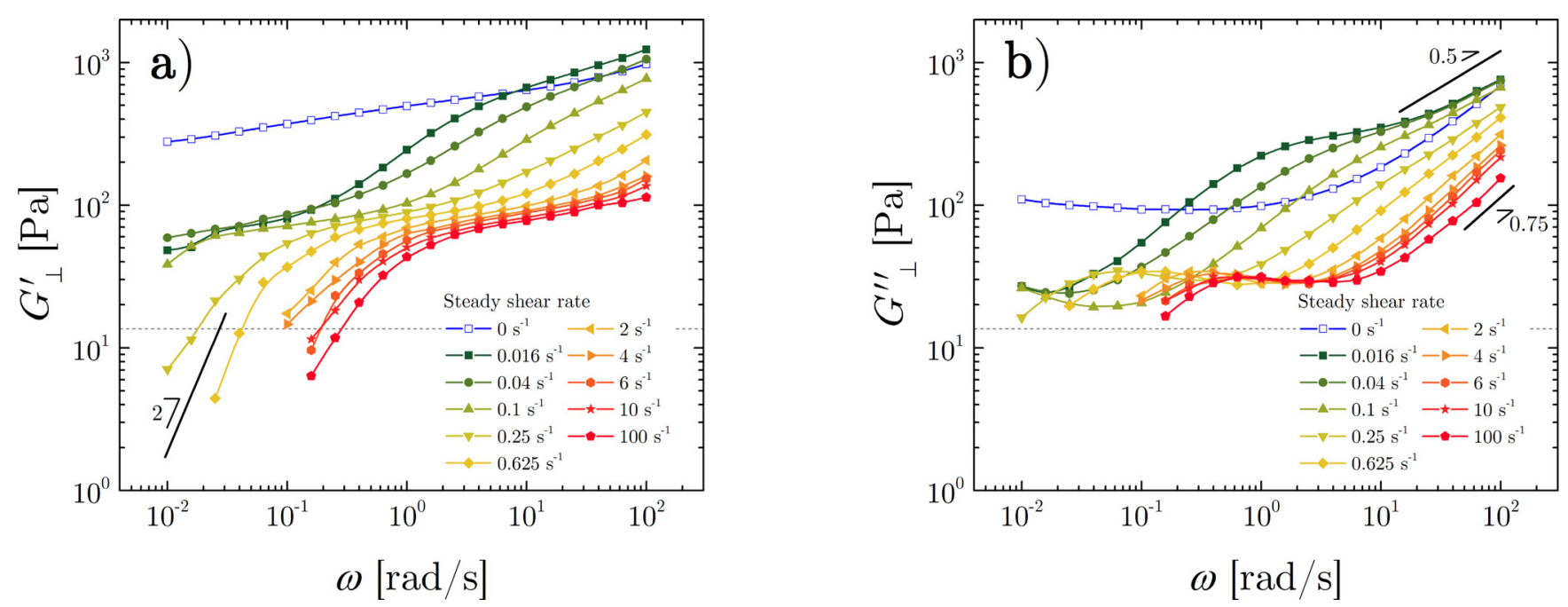

FIG. 3. Orthogonal superposition moduli $G_{\perp}^{\prime}$ (a) and $G_{\perp}^{\prime \prime}$ (b) as a function of frequency at a strain amplitude $\gamma_{0}=0.22 \%$, for different shear rates of the main flow. The low-force resolution limit in oscillation of the instrument is shown by the dashed line. A slope of 2 for the storage modulus as expected for terminal behavior is included for comparison, as well as slopes of 0.5 and 0.75 for the loss modulus. Solid lines are to guide the eye.

together with the data for a reference structure (denoted in

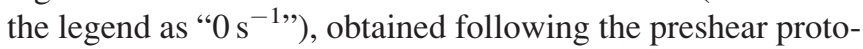
col and allowing for structural recovery for $5400 \mathrm{~s}$. It has to be mentioned that this reference structure is not the absolute rest structure, which is never completely attained. It is, therefore, a somewhat arbitrary, but reproducible structure produced by the preshear and subsequent recovery protocol, and then measured in OSR with no steady shear flow applied. The response of such structure represents a typical example of what would be obtained for a solid, percolating material, with physical interparticle bonds and a dominant elastic response. A weak power law regime is observed for the storage modulus, possibly stemming from the fractal nature of this rather strongly aggregating physical gel, as expected for the investigated particle volume fraction and the preshear protocol employed.

As shear flow is applied, both the magnitude and characteristic shape of $G_{\perp}^{\prime}$ and $G_{\perp}^{\prime \prime}$ versus $\omega$ change (Fig. 3). For the lowest shear rates, the superposition moduli at high frequency increase above those of the reference structure. For hard spheres, the high frequency regime is reached when the thickness of diffusion and lubrication boundary layers becomes similar [5]. This corresponds to $\omega \gg k_{B} T / 6 a^{3} \pi \eta_{m}$, which is expected to be around $10 \mathrm{rad} / \mathrm{s}$ for the primary aggregate sizes and the medium viscosities used. Theoretical predictions of Lionberger and Russel for hard sphere suspensions in the free draining limit produce a scaling of the elastic modulus with frequency to the power of 0.5 [5], as opposed to a plateau in the moduli that would be reached for soft interaction potentials or when hydrodynamics dominate and the lubrication forces dictate the response. This predicted hard sphere scaling was for instance observed by Fritz et al. [43]. Varga and Swan [6] showed how the expected high frequency hard sphere response is modulated by the presence of attractive interactions, due to changes in the local density distribution. The latter may be further modified during flow by additional flow-induced aggregation and compaction on local length scales [17,21,22]. Varga and Swan [6] showed that strong attractions lead to a delay in the onset of a high frequency, power law scaling and to an intermediate elastic plateau. Although we only access part of the high frequency regime, we observe that the power law behavior of both high frequency moduli varies between exponents of 0.5 and 0.75 . To fully attain the high frequency regime, alternative approaches using piezo based rheometers or resonators are necessary. Yet, qualitatively, a behavior more akin to stable dispersions is observed at higher shear rates, while at lower shear rates the attractions and the corresponding larger aggregates shift the high frequency regime to higher frequencies, with only a weak dependency of the power law on the shear rate. In the present systems at low volume fraction, the high frequency behavior can only reflect changes in the viscoelastic properties of the aggregates, which make up the network, in contrast to more concentrated systems like hard sphere glasses [44], where caging introduces additional short time dynamics.

At low frequencies, the effects of the main shear rate are more pronounced. The moduli during flow drop significantly, reaching a steep-sloped regime for shear rates of the main flow starting from $\dot{\gamma}=0.25 \mathrm{~s}^{-1}$. This indicates that connectivity is lost, in agreement with stress jump measurements [45], which show that from around this shear rate the elastic contribution to the stress starts to decrease. Also, in the structural kinetic modeling of the same system [46], the structural parameter $\lambda$ decreases and becomes smaller than 1 , the value corresponding to a fully developed structure. As the shear rate is increased, the magnitude of the superposition moduli decreases at all frequencies, even at those larger than the inverse of the characteristic time scale of flow $\dot{\gamma}^{-1}$. This is quite different from the response observed in polymeric materials [47] or colloidal glasses [44], where convective motion cuts off the relaxation spectrum. Earlier measurements by Schoukens and Mewis for carbon black suspensions showed only power laws for the storage moduli versus frequency over the accessible frequency range [48].

At intermediate frequencies, a near-plateau region can be observed, which persists up to high shear rates. There, the steady state stresses are then much higher than the dynamic 
yield stress (Fig. 1). This is in contrast to results of stress jump experiments on similar materials in which elasticity decays much faster with shear rate [45]. In the pseudoNewtonian regime, the viscous stress is expected to dominate, and the stress jump experiments confirm this. In the latter, however, the response of the shear stress component (in the $\theta-r$ plane) is reported, and the loss of elasticity and structure is more dramatic than for the superposition experiments (which probe the $\theta-z$ plane), as will be illustrated by the measurements upon cessation of flow presented below. The curves of the moduli versus frequency evolve very gradually with increasing shear rate, mainly shifting to higher frequencies. Comparing the observed viscoelasticity with a plateau modulus in the orthogonal superposition in this system with the scattering experiments on a wide variety of systems, where butterfly scattering patterns are observed [17,26], suggests that the viscoelastic response is due to large structures orthogonal to the main flow direction. These structures remain present even when the shear stress response is dominated by purely viscous stresses (see Fig. 1). The very gradual evolution of the plateau at intermediate frequencies suggests that the structural length scales in the vorticity direction also break down gradually. In the absence of direct structural measurements, it is not clear whether the inherent elasticity of porous, fractal aggregates, [9] or the Brownian motion of dense isostatic clusters [14] is responsible for the observed plateau; yet, the gradual shift implies that the aggregate structures in the velocity-vorticity plane only gradually erode. Similar features were observed for depletion gels, where direct structure measurements showed that the contact number distribution changes with the strain in a way that is consistent with the erosion of rigid clusters [15].

Further insight can be gained by inspecting the phase angle data under flow, shown in Fig. 4. Again, the distinction between plastic flow and viscous flow regime can be clearly identified. At low shear rates, the low frequency phase angle is consistent with dominant elastic behavior. All curves show an upturn in loss angle at the lowest frequencies,

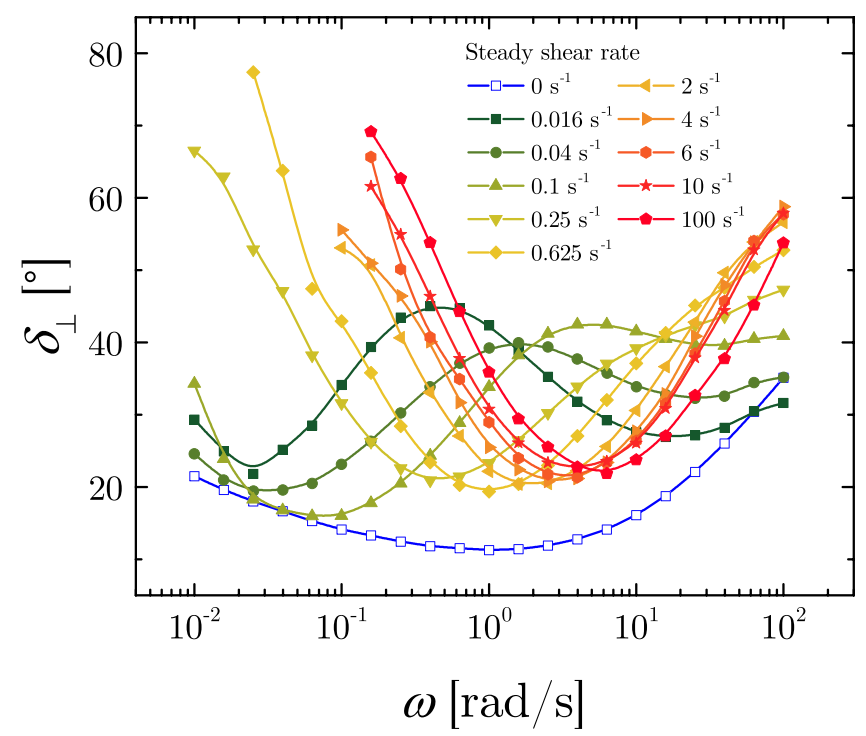

FIG. 4. Orthogonal superposition phase angle $\delta_{\perp}$ at a strain amplitude $\gamma_{0}=0.22 \%$. The main flow shear rate is indicated in the legend. characteristic for a system with physical bonds. The loss angle, starting from $\dot{\gamma}=0.25 \mathrm{~s}^{-1}$, abruptly increases, suggesting the loss of (transient) connectivity upon yielding with increasing shear rate. The high frequency behavior should be related to the individual flocs, as argued above. Because of the two relaxation mechanisms, at higher rates, a clear minimum appears in the phase angle, which shifts to higher frequencies with increasing shear rate, as shown more clearly in Fig. 6(c). This trend reflects how flow affects the equilibrium aggregate size, which decreases as shear becomes more intense. However, in agreement with the observations at intermediate frequencies for $G_{\perp}^{\prime}$, this process causes a minimal vertical shift in phase angle at the minimum in the flow regime. This reflects a gradual erosion of the aggregates. Plotting the phase angle versus the complex modulus, in a so-called Van Gurp-Palmen plot, collapses the curves with $\dot{\gamma} \geq 0.25 \mathrm{~s}^{-1}$, as is shown in Fig. 5 .

Such a collapse into a single curve is usually observed in polymeric systems obeying the time-temperature superposition principle. Therefore, Fig. 5 suggests an interesting analogy in the form of a "time-shear" superposition, where shear flow changes the relaxation time of the aggregates in a rather simple way, by progressively reducing their size without changing the internal structure, at least for deformations in the $\theta-z$ plane and from a shear rate of $0.25 \mathrm{~s}^{-1}$ onward. This gradual erosion is consistent with existing microrheological models for weakly aggregated dispersions under shear [13] and with the observations of the existence of flow regime butterfly scattering patterns. As expected, such simple description fails in the plastic flow regime. There, the interplay between shear and the microstructure will be more complex, as no well defined aggregates are formed yet, so that a vertical shift is also observed. Also, a gradual loss of overlap between the curves is observed at higher values of $G^{*}$, i.e., at higher frequencies, capturing the increasing amount of structure as the shear rate is decreased.

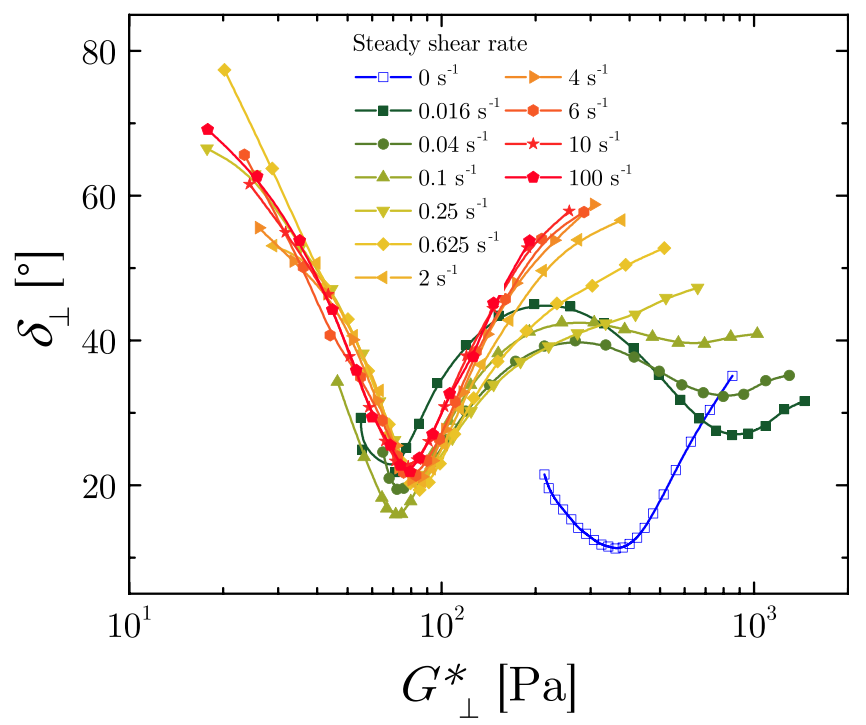

FIG. 5. Orthogonal superposition phase angle $\delta_{\perp}$ (left) versus superposition complex modulus $G_{\perp}^{*}$ at a strain amplitude $\gamma_{0}=0.22 \%$. The main flow shear rate is indicated in the legend. 

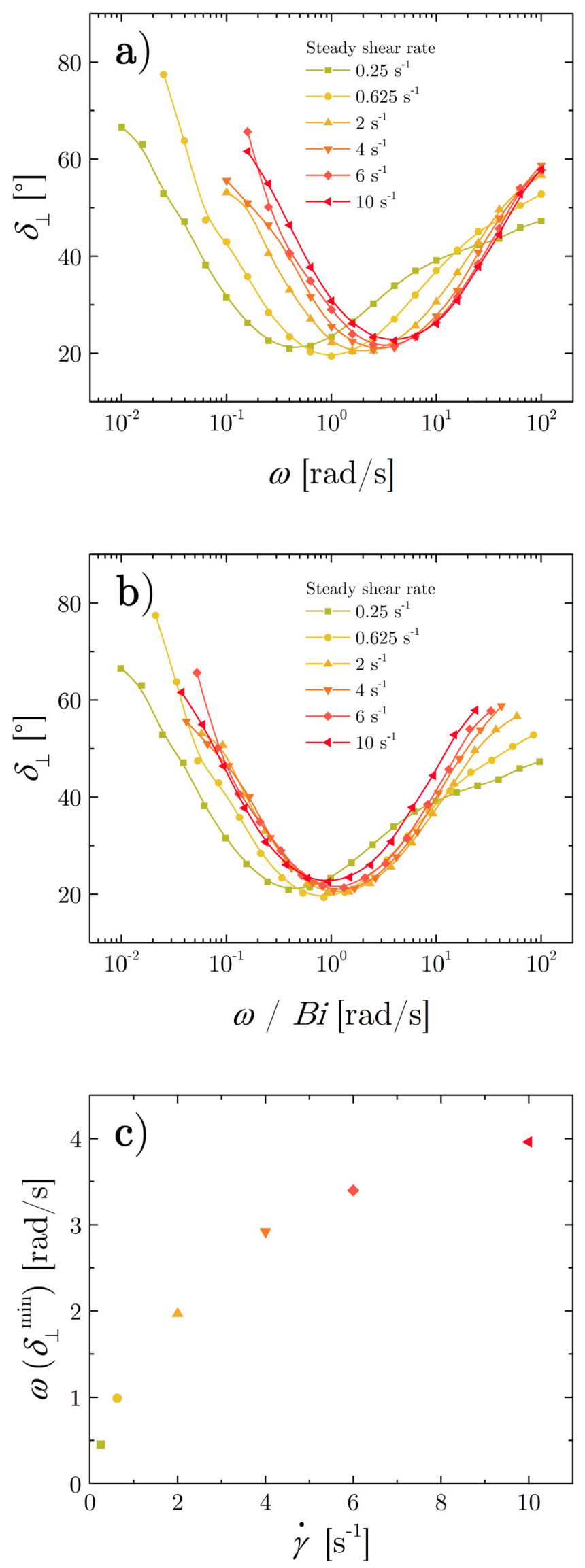

FIG. 6. Orthogonal superposition phase angle $\delta_{\perp}$ versus unscaled (a) and scaled (b) frequency range $\omega$, for a selection of shear rates. The intensity of the steady flow is indicated. The frequency at the minimum of (a) is plotted in (c) against the main flow shear rate $\dot{\gamma}$.
Given the mechanism of gradual aggregate size reduction with increasing shear rate hypothesized above, it should then be possible to collapse the phase angle data also using the characteristics of the steady shear flow, at least for the curves in the flow regime. A physically sound choice is the Bingham number, a dimensionless group defined here as $B i=\sigma / \sigma_{y}$. The Bingham number compares the hydrodynamic stresses to the structural stresses, which are due to the attractive interactions, through the yield stress. The latter is proportional to the maximal attractive interparticle force, i.e., the maximum slope of the pair potential $\Phi: \sigma_{y} \sim$ $\phi^{2} / a^{2}(d \Phi / d r)[49,50]$. This scaling proved successful for the steady shear rheology of thermoreversible octadecyl silica gels [17]. Figure 6 shows the result of such scaling for a selection of shear rates from Fig. 4, corresponding to a Bingham number range $1<B i<4.5$. The frequencies at the minimum in phase angle of Fig. 6(a) are plotted against the shear rate in Fig. 6(c).

The proposed scaling is able to capture the shift in frequency upon flow in the expected range of shear rates, corresponding to stresses beyond the plastic flow regime (the shear rate of $0.25 \mathrm{~s}^{-1}$ being at the edge of the latter, hence not scaling so well). The scaling, therefore, holds (at least approximately) not only for more homogeneous systems such as the thermoreversible gels studied by Wagner and coworkers $[17,49]$ but also for the present, more heterogeneous colloidal gel. Therefore, the emerging picture is that the aggregates gradually evolve and decrease in size during more intense shearing, as a result of the interplay between the shear forces breaking them up and the maximum attractive interparticle force. As the shear rate is increased, the position of the minimum in phase angle moves more slowly to higher frequencies, indicating how the aggregates turn more resistant to further erosion as their size is reduced by the flow.

The emergence of the observed minima in superposition phase angle could be due to the slow Brownian relaxation of aggregates. This hypothesis has been presented in the literature to explain significant elastic responses in colloidal gels under flow [14]. The consistency of this hypothesis with the present measurements can be tested by estimating an equilibrium aggregate hydrodynamic size, using the frequency at the minimum in the unshifted phase angle data of Fig. 4 as inverse characteristic Brownian diffusion time $\tau_{D}=L^{2} / D$. This is, therefore, calculated under the assumption that Brownian motion is responsible for the observed viscoelasticity of the material. The characteristic length $L$ is taken as the size of the aggregate itself, and the Stokes-Einstein relation is used with the medium viscosity and the diffusion coefficient $D$ to estimate a typical equivalent hydrodynamic radius. The obtained size ranges from a radius $a=195 \mathrm{~nm}$ for a shear rate of $0.25 \mathrm{~s}^{-1}$, down to $85 \mathrm{~nm}$ for the highest shear rate of $100 \mathrm{~s}^{-1}$. The obtained hydrodynamic radii values are consistent with the expected size range, given the elementary particle average radius of $14 \mathrm{~nm}$ [51] and the size of their dense agglomerates of about $100 \mathrm{~nm}$, estimated by $\mathrm{X}$-ray scattering [52]. However, lacking direct structural information, it still cannot be concluded unequivocally that the observed elasticity and the minimum in superposition 

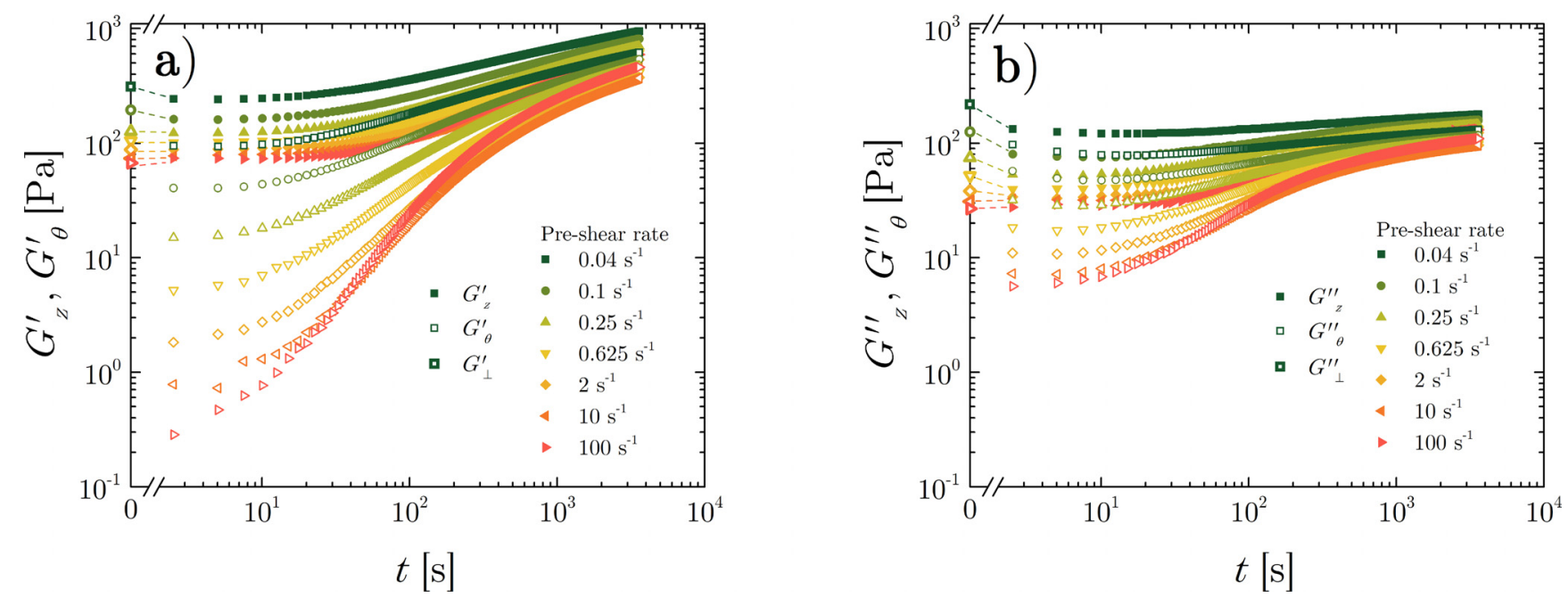

FIG. 7. Storage (a) and loss (b) moduli upon cessation of flow in orthogonal $\left(G_{z}^{\prime}\right.$ and $G_{z}^{\prime \prime}$, filled symbols) and parallel $\left(G_{\theta}^{\prime}\right.$ and $G_{\theta}^{\prime \prime}$, open symbols) direction with respect to the previous flow. The symbols on the $y$-axis, at $0 \mathrm{~s}$, indicate the values obtained at the corresponding frequency (5 rad/s) during flow $\left(G_{\perp}^{\prime}\right.$ and $G_{\perp}^{\prime \prime}$, Fig. 3). The dashed lines are to guide the eye. The intensity of the main flow before an arrest is indicated.

phase angle, are indeed due to the Brownian relaxation of dense aggregates, as stated by Hsiao et al. for their system $[14,15]$. Other contributions coming from, e.g., the poroelastic breathing modes of the aggregates themselves could be contributing in this frequency regime.

For investigating mechanical anisotropy, the orthogonal moduli have to be supplemented with similar measurements in another direction, e.g., parallel to the main flow. In many commercial devices, such parallel oscillatory flow can be superimposed on the steady shear flow. However, interpretation of such data remains difficult due to the coupling between the main flow and the superimposed perturbation flow, as discussed earlier [18]. Yet, superposition flows become ordinary oscillatory flows as soon as the steady shear is arrested. Here, we use a thixotropic system where structure and properties recover gradually after arresting the flow. The evolution of the mechanical anisotropy from steady shear flow to equilibrium rest conditions can then be univocally followed without any interference from the main flow. Figure 7 compares the time evolution of the storage and loss moduli upon cessation of flow, measured in mutually perpendicular directions, for values of the prior shear rate similar to the range explored in Fig. 3. A relatively high frequency of 5 $\mathrm{rad} / \mathrm{s}$ was used to allow for fast enough sampling.

The open symbols show the evolution of the storage moduli parallel to the previous flow direction, indicated with the subscript $\theta$. Such time evolution after cessation of flow of what are typically called "the" moduli was reported for many types of colloidal gels, with an increase of orders of magnitude in storage modulus [37,53-57]. In comparison, the loss moduli are far less affected by flow. The storage moduli $G_{z}^{\prime}$, measured in the orthogonal direction, i.e., perpendicular to the previous flow direction, show a much weaker dependence on shear rate, as well as a significantly larger magnitude. This is a consequence of the previous shear flow selectively breaking down the microstructure in the velocity-velocity gradient plane, while affecting it much less in the velocity-vorticity plane, in the neutral direction of shear, where no direct forces are present, in agreement with scattering and microscopy studies $[17,22,26]$. The resulting anisotropy, expressed as the ratio of the storage moduli in the two directions, is substantial, as shown in Fig. 8. The good agreement between steady state data in orthogonal superposition flows (indicated with open symbols at $0 \mathrm{~s}$ in Fig. 7) with the initial values of the recovery curves confirms that the orthogonal oscillation is sufficiently decoupled from the main flow, so that the superposition moduli have the same physical meaning as ordinary linear moduli. Some fast relaxation mechanisms, also observed in stress jump measurements [45], may introduce minor discrepancies. However, it is clear that the OSR measurements probe the most dominant aspects of the structural evolution under flow.

For the lower shear rates up to $0.1 \mathrm{~s}^{-1}$, the storage moduli in the two directions initially differ by a factor of $2-4$, up to a difference of 2 orders of magnitude at the highest shear rates. As could be seen already in Fig. 7, the anisotropy in

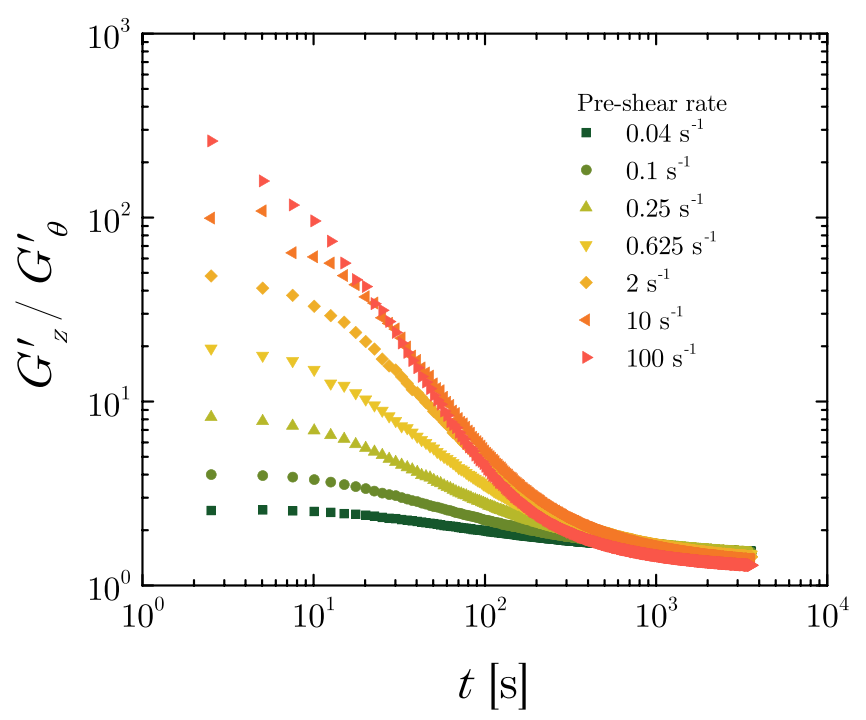

FIG. 8. Mechanical anisotropy expressed as the ratio of storage moduli in perpendicular directions, upon cessation of flow. The intensity of the main flow before an arrest is indicated. 
loss modulus is smaller by about an order of magnitude. Importantly, the microstructural anisotropy subsides slowly upon cessation of flow, at the same long timescale of the thixotropic build-up. Figure 8 shows how the anisotropy gradually decreases, as the microstructure evolves back to an isotropic state. The persistence of the anisotropy further suggests that selective breakdown rather than shear-induced orientation of the microstructural features is occurring.

A similar mechanism of a spatially selective breakdown was hypothesized for nonaqueous layered silicate suspensions [19], where mechanical anisotropy in a polymer-clay nanocomposite was measured for the first time, with differences of the moduli in orthogonal directions of a factor 5 . For the fumed silica suspensions studied here, which are formulated at a similar volume fraction, the degree of anisotropy is significantly higher, by almost 2 orders of magnitude. In the layered silicate suspensions, the interplay between a possible flow-induced orientation of the platelets composing the gel and the anisotropic network breakdown could lower the observed anisotropic response, as these two effects would affect the degree of anisotropy in opposite directions. In contrast, for the present measurements, the anisotropy can be attributed solely to the spatially selective breakdown of the network structure, as the fumed silica particles and their agglomerates are essentially isotropic.

The fractal, isotropic nature of the agglomerates in the fumed silica suspensions, referred to here as the primary aggregates of partially coalescing primary particles, arises from the conditions experienced by the growing silica particles during the flame synthesis and was proved by electron microscopy and scattering experiments [51,58]. Primary, spherical particles emerge from the initial, hotter part of the flame, while the strongly bound agglomerates stem from collisions and partial coalescence of the primary particles in the following cooler regions of the flame. In later stages, larger flocs are obtained, which are, however, only resulting from physical aggregation. The same fumed silica used in this work was found to scatter X-rays with a power law exponent of -3 , when dispersed in a similar matrix and using a similar mixing protocol, thus indicating very dense, near spherical agglomerates [52].

For suspensions in thermosetting or thermoplastic matrices, the anisotropic microstructure could be locked in to create novel functional soft materials with anisotropic mechanical, thermal or optical properties. Figure 8 indicates how both shear rate and time upon cessation of flow could be used to tune the properties of such materials.

It is insightful to compare the present results with those of other material classes, for example with liquid crystalline polymers. In simple shear flow, the mechanical anisotropy of our gel is more than 1 order of magnitude larger than that of lyotropic liquid crystals probed in the same way [59]. A second comparison can be made with dense suspensions of non-Brownian spheres, in the limit of a high Péclet number. Stokesian dynamics simulations show for this case a maximal anisotropy of a factor of order 2, as quantified by the asymmetry of the radial distribution function [60].

It should be remarked that the anisotropy does not reach the expected final value of 1 in Fig. 8. This is an artifact of the measurement caused by the residual pumping flow in the orthogonal measurement discussed above, leading to an increased strain amplitude in the axial direction. This is a problem especially for systems with a yield stress as discussed earlier. As the sample is thixotropic, the magnitude of the offset now depends on the applied shear rate. The preshear rate determines the extent of liquefaction the sample undergoes before the recovery measurement is started. Therefore, for low preshear rates, the axial motion is not necessarily strong enough to push material into the central reservoir through the bottom openings. The resulting backflow in the measuring gap, which also increases as the gel microstructure recovers, is reflected in an overestimation of $G_{z}^{\prime}$. This fact explains the crossing of the curves in Fig. 8. However, numerical calculations on a Newtonian fluid (see the Appendix) show that for a true solid wall at the openings, the resulting pressure force and Poiseuille flow would dominate the simple shear, leading to a much larger offset (almost 2 orders of magnitude) than the ca. $+50 \%$ actually observed for the lowest applied shear rate.

To investigate the anisotropy further, we used 2D-SAOS measurements, where a bidirectional flow field is imposed by coupling the axial deformation $\gamma_{z}=\gamma_{z}^{0} \sin (\omega t)$ with the conventional angular deformation $\gamma_{\theta}=\gamma_{\theta}^{0} \sin \left(\omega t+\delta_{z, \theta}\right)$ [19]. With a phase offset $\delta_{z, \theta}=0^{\circ}$, a unidirectional, screwlike deformation is obtained, whose orientation angle in the velocity-vorticity plane of the main shear flow can be tuned by varying the relative magnitude of the strain in the two directions $\varphi_{z, \theta}=\arctan \left(\gamma_{z}^{0} / \gamma_{\theta}^{0}\right)$. The resulting peak amplitude is given by $\sqrt{\gamma_{z}^{0^{2}}+\gamma_{\theta}^{0^{2}}}$, the stress response is then calculated in the same way from the components in $z$ and $\theta$ direction, and its harmonic analysis leads to the viscoelastic moduli at the given angle $\varphi_{z, \theta}$. The results of a recovery experiment analogous to those in Fig. 7 are shown for a preshear rate of $10 \mathrm{~s}^{-1}$ in Fig. 9. Again, a frequency of $5 \mathrm{rad} / \mathrm{s}$ was used to ensure adequate sampling rates.

The limiting cases of vorticity and flow direction, for $90^{\circ}$ and $0^{\circ}$, correspond to the experiments shown in Fig. 7 for the orthogonal and parallel case, respectively. Interestingly, it can be seen that already at a small angle of $22.5^{\circ}$ from the flow direction, most of the difference between flow and vorticity direction is recovered, while the curves for $67.5^{\circ}$ and $90^{\circ}$ are almost overlapping. This observation strengthens the conclusion that the measured mechanical anisotropy is due to the spatially selective breakdown of the microstructure in the direction of the previous shear flow. Again, the storage moduli are much more affected by the previous shear than the loss moduli.

The dispersion quality of the samples is influenced by mainly two factors: The used mixing procedure and the physical aging processes occurring over long times. Both factors affect the microstructure, effectively changing the recovery behavior of the sample and the observed anisotropy. A less effective dispersion procedure results in both a lower degree of anisotropy and a faster recovery to an isotropic structure, as shown in Fig. 10. In this case (denoted as "mild mixing"), the silica powder was dispersed as described 

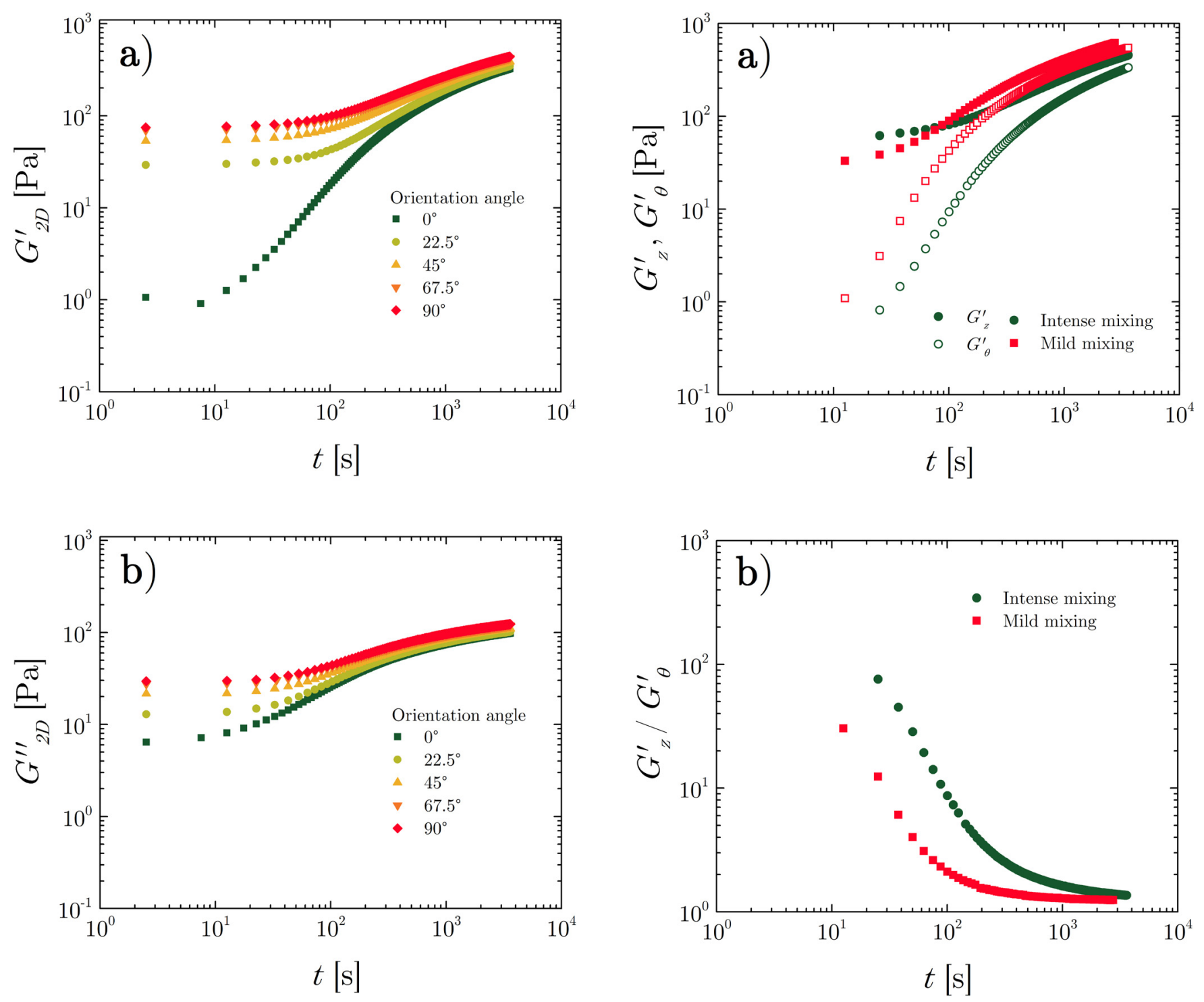

FIG. 9. 2D storage (a) and loss (b) moduli $G_{2 D}^{\prime}$ and $G_{2 D}^{\prime \prime}$ upon cessation of steady shear flow at $10 \mathrm{~s}^{-1}$. The orientation angle $\varphi_{z, \theta}$ of the $2 \mathrm{D}$, unidirectional deformation is indicated.

in Sec. II, but the suspension was subjected to ultrasonication only after the last addition step.

The results in Fig. 10 confirm the crucial role of dispersion quality on the rheology of the present model system. The storage moduli measured in the direction parallel to the previous flow are more susceptible to breakdown under shear than the orthogonal ones, and therefore also more sensitive to the quality of dispersion. In turn, the orthogonal storage moduli even cross, so that a sample with lower dispersion quality recovers more quickly. This results in a decreased and faster fading anisotropy. Aging produces results similar to those shown for mixing in Fig. 10.

Many soft materials demonstrate anisotropic rheological properties, often related to either an inherent anisotropy of the building blocks, as in liquid crystals [59] or due to a field-directed assembly of the microstructure, as in magnetorheological fluids [61]. However, as recently pointed out by Cohen and coworkers [62], an anisotropic microstructure does not automatically lead to an anisotropic rheological

FIG. 10. Comparison of two identical formulations, prepared with different mixing procedures. Storage moduli in vorticity $\left(G_{z}^{\prime}\right.$, filled symbols) and flow $\left(G_{\theta}^{\prime}\right.$, open symbols) direction, upon cessation of the steady shear flow (a). The corresponding anisotropy as the ratio of the storage moduli in the two perpendicular directions (b). Measurements at angular frequency $\omega=1 \mathrm{rad} / \mathrm{s}$ and strain amplitude $\gamma_{0}=0.22 \%$, upon cessation of steady shear flow at $10 \mathrm{~s}^{-1}$.

response. The latter authors investigated a suspension of colloidal spheres which formed aligned string phases during shear, but no significant anisotropy in the viscous response was detected. Also, Stokesian dynamics simulations showed that shear thinning of the viscosity is decoupled from the layering of a stable colloidal suspension under shear [63]. For the flocculated suspensions investigated here, the loss moduli also show a weaker dependence on shear rate, and hence on the structural anisotropy, suggesting that the hydrodynamic contributions to the stress do not depend too strongly on the microstructure. These can still be expected to vary because of changes in porosity of the network or changes in size, density, and effective volume fraction of the constituent aggregates. Yet, unexpectedly for these materials, the elastic properties show a very high degree of anisotropy. This suggests a strong difference in load bearing 
structures in the two directions, consistent with the microstructural observations by either scattering techniques or direct observations. The structure in the vorticity direction can be expected to be affected only weakly by flow, as there are no direct forces acting in what is appropriately called the neutral direction. This is confirmed by the comparatively weak dependence of the moduli in the orthogonal direction on shear rate (Fig. 7).

In the direction of straining the situation is known to be different, as evidenced by microstructural observations. At low stresses, close to the yield point, the flocculated suspension flows, while maintaining a transient network $[21,25,26]$, which is observed to be anisotropic, with load bearing structures forming along the compressional axis of the flow field. Interestingly, the anisotropy in the mechanical response is preserved even when connectivity in the microstructure can be expected to be lost, based on the flow curve. Some significant elasticity is retained, even when the system enters the pseudo-Newtonian regime in Fig. 1 and the viscous response becomes dominant (Figs. 3 and 4).

The results of the cessation of flow experiments shed new light on the obtained orthogonal superposition moduli, which are measured in the same direction where a largely unaffected structure is found after stopping the previous shear (Fig. 7). It has to be pointed out that the cessation of flow experiments are carried out at $5 \mathrm{rad} / \mathrm{s}$, a choice motivated by the trade-off between time resolution after flow arrest and frequency. This relatively high frequency is in the range, where a relaxation time is probed which corresponds to more local features at the aggregate scale, rather than that of the network. Therefore, long range connectivity is also lost in the orthogonal direction, as evidenced by the sharp increase of the superposition phase angle at low frequencies in Fig. 4, but substantial elasticity is retained at smaller length scales, which also reflects in the highly anisotropic structure observed after flow cessation for this frequency. Given the relaxation time probed, this should be due to anisotropic, elongated aggregates remaining in the direction orthogonal to previous shear flow. The recent idea that rigid, isostatic clusters with a viscoelastic Brownian time scale contribute is interesting to investigate [14] and consistent with the above presented data. Yet, when connecting the flowinduced microstructure of these types of soft materials to their nonlinear rheology, the anisotropic microstructure is an essential or even crucial element, especially when important nonhydrodynamic contributions are present.

\section{CONCLUSION}

OSR and 2D-SAOS were employed to study shear-induced microstructural changes and mechanical anisotropy in a model thixotropic gel. The orthogonal superposition moduli, obtained during steady state flow at different shear rates, provide direct frequency-dependent information about the effects of shear rate on flocculated suspensions. Two main relaxation processes relate to the (residual/transient) network relaxation at low frequencies and to a fast relaxation of the aggregates, respectively. Low shear rates, with stresses very close to the dynamic yield stress, already drastically affect the superposition moduli and the relaxation mechanisms, in particular at low frequencies. The pseudoplateau region of the storage moduli, characteristic for the physical gel that exists at rest, drops drastically at even the smallest shear rates, consistent with a gradual loss of connectivity, leading to a transient network and to breakdown into systematically smaller structures. However, when the shear rate is further increased, a lower plateau persists even at shear rates corresponding to the pseudo-Newtonian part of the flow curve. Increasing the shear rate reduces the relaxation time of the aggregates, but the near-constant level of the plateau in the moduli suggests structural elements that can still relax in the same manner. A mechanism of erosion without significant internal structural changes can be inferred from the Van Gurp-Palmen plot. The flow regime phase angle curves also collapse in a frequency range scaled by the Bingham number of the main flow.

Additional information was obtained during structural recovery by means of oscillatory measurements in the flow direction and by 2D-SAOS. During steady flow, the parallel superposition flow cannot be analyzed as normal linear oscillatory flow. This difficulty disappears after the flow has been arrested. In the thixotropic systems used here, the evolution in time of parallel and orthogonal moduli could be compared after arresting the flow. An initial difference of up to 2 orders of magnitude between the storage moduli in the two directions was measured, significantly higher than those observed in layered silica-polymer systems studied by Mobuchon et al. [19], where such anisotropies were reported for the first time. Hence, flow induces a very large anisotropy in these materials that cannot be directly associated with the shape of the elementary particles. The difference is less pronounced for the loss moduli. For both types of moduli, the initial anisotropy increases with the shear rate over the range covered here and decays slowly when the structure recovers in time. The data are congruent with earlier microstructural studies by scattering and direct observations on similar systems. Upon yielding, the present gel breaks down in a spatially anisotropic way. Bonds in the velocity-velocity gradient plane break down, whereas microstructural features are less affected away from this plane. The rheological data demonstrate that anisotropy cannot be ignored in understanding and modeling flow in these weakly flocculated and gelling suspensions. It is also shown that the detailed results are very sensitive to the details of the dispersion process.

\section{ACKNOWLEDGMENTS}

The authors thank Jan Hendricks for help with the initial OSR measurements in Leuven. G.C. and J.V. acknowledge support of the Swiss National Science Foundation, Project No. 157147. S.K. and C.C. acknowledge support from the ERC starting Grant No. 203043 - NANOFIB.

\section{APPENDIX: ESTIMATION OF MEASUREMENT ERRORS DUE TO PUMPING FLOW}

The flow profiles of a Newtonian fluid in the orthogonal direction of the orthogonal superposition setup were modeled by CFD simulations in COMSOL multiphysics. Two limiting 


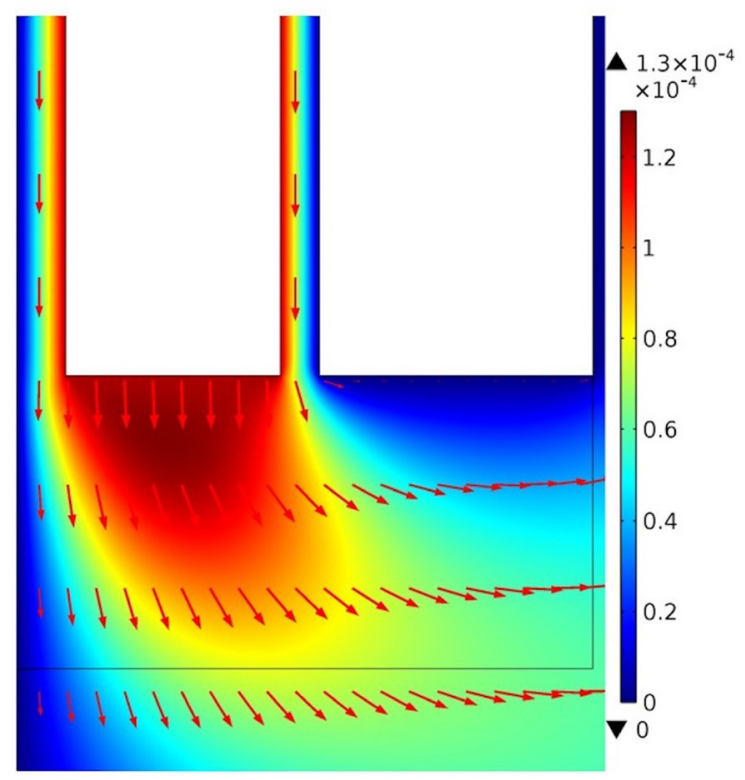

a)
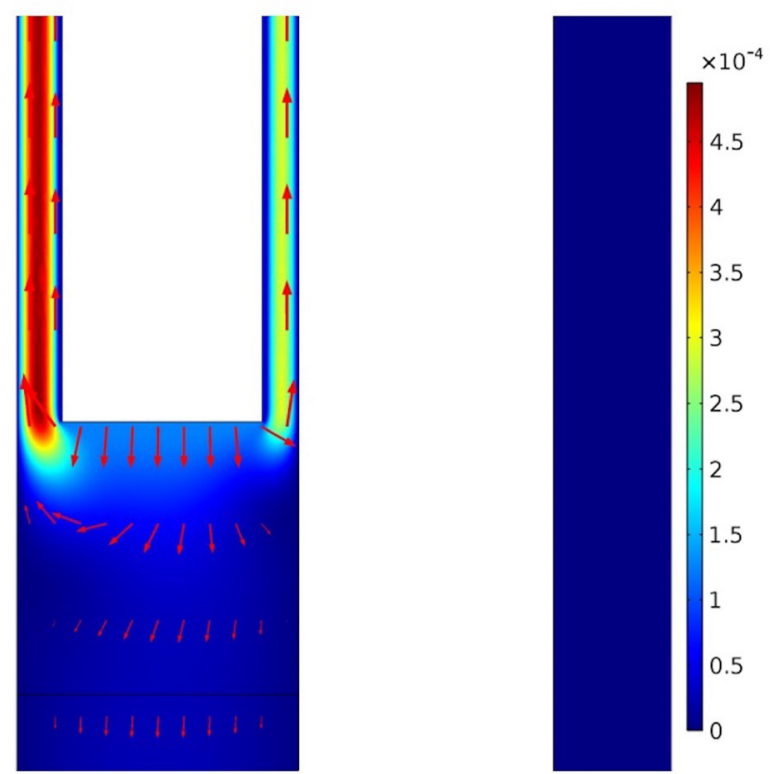

b)

FIG. 11. Velocity magnitude (m/s_color/greyscale) and velocity field (arrows) for the open (a) and closed (b) cup bottom. The bottom of the cell is shown to emphasize the outflow from or backflow into the measuring gaps. The bob is moving downward at a velocity of $1.25 \times 10^{-4} \mathrm{~m} / \mathrm{s}$.

cases of openings and a solid wall at the inner side of the cup were considered, using 2D calculations of the velocity fields. In addition, the behavior was simulated in $3 \mathrm{D}$ for the open bottom case, to check the effects of the supporting pillars of the cup. The two considered limiting cases represent, respectively, a lower and an upper bound for the overestimation of the superposition moduli of a yield stress fluid in OSR. The yield stress fluid in the openings at the measuring cell bottom undergoes a different level of liquefaction depending on the experienced shear, resulting in different amounts of error due to pumping flow, as discussed in Sec. III.

The laminar flow interface was used to simulate the fluid behavior during oscillatory flow. This interface couples the Navier-Stokes equations with the continuity equation to solve for both the pressure and velocity field. Simulations were performed on a noncompressible fluid with a viscosity of $1 \mathrm{~Pa}$ s. A no-slip boundary condition was applied at the walls of the bob and cup, whereas the fluid/air boundary was considered to be a stress free boundary. The bob was oscillated in the orthogonal direction with a frequency of $5 \mathrm{rad} / \mathrm{s}$ using a strain amplitude of $5 \%$.

The resulting velocity fields and profiles over the measurement gaps are shown in Fig. 11 for the 2D models. For the Newtonian case, they are qualitatively similar to the $3 \mathrm{D}$ case (where a coarser mesh had to be used). A clear difference can be seen from the velocity magnitude in the gap between both limiting cases, which is shown more in detail in Fig. 12, via the orthogonal velocity profile over the outer measurement gap. There the difference between the nearly linear shear profile and Poiseuille dominated flow for the closed wall case is apparent.

The effect of pumping flow is quantified for both limiting cases by calculating the forces acting on the bob when the predefined oscillatory deformation is applied. The results are shown in Table I. They are calculated by integrating the local forces over the entire geometry. The total force consists of contributions of the force acting on the side walls (viscous) and ends of the bob (pressure). As for the velocity fields, the results for the $2 \mathrm{D}$ and $3 \mathrm{D}$ models in the open bottom case are nearly identical, showing the limited effect on both force contributions of the pillars between the bottom openings. The moduli are calculated from the force using the geometrical parameters of the setup. For the open bottom case, the calculated modulus is overestimated by approximately $10 \%$. The offset for this lower bound is caused by a combination of an increased shear rate in the gap as a result of a limited amount of pumping flow, together with pressure forces on the bob ends. For the closed bottom case, both force

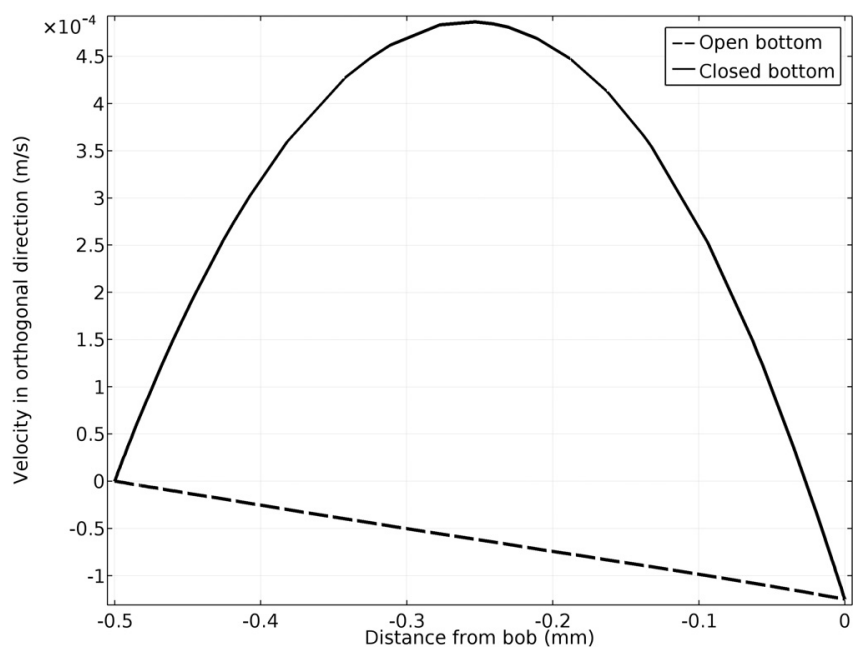

FIG. 12. Velocity profile in the orthogonal direction over the outer measurement gap. The moving bob wall is the reference position at $0 \mathrm{~mm}$. 
TABLE I. Comparison of the CFD simulations results for the experimentally observable moduli and viscosities with the theoretical values, for either open or closed bottom of the cell cup.

\begin{tabular}{lcccc}
\hline \hline & 3D-open & 2D-open & 2D-closed & Theoretical value \\
\hline Viscous contribution & $2.57 \mathrm{mN}$ & $2.55 \mathrm{mN}$ & $38.3 \mathrm{mN}$ & $2.5 \mathrm{mN}$ \\
Pressure contribution & $0.19 \mathrm{mN}$ & $0.11 \mathrm{mN}$ & $177 \mathrm{mN}$ & \\
Total force & $2.76 \mathrm{mN}$ & $2.65 \mathrm{mN}$ & $215 \mathrm{mN}$ & \\
Calculated modulus & $5.52 \mathrm{~Pa}$ & & $430 \mathrm{~Pa}$ & $5 \mathrm{~Pa}$ \\
Calculated viscosity & $1.1 \mathrm{~Pa} \mathrm{~s}$ & & $86 \mathrm{~Pa} \mathrm{~s}$ & $1 \mathrm{~Pa} \mathrm{~s}$ \\
\hline \hline
\end{tabular}

contributions increase markedly, but the response is now dominated by the pressure contribution. The calculated modulus is overestimated by almost 2 orders of magnitude.

\section{References}

[1] Smay, J. E., G. M. Gratson, R. F. Shepherd, J. I. Cesarano, and J. A. Lewis, "Directed colloidal assembly of 3D periodic structures," Adv. Mater. 14, 1279-1283 (2002).

[2] Wang, Q., L. Wang, M. S. Detamore, and C. Berkland, "Biodegradable colloidal gels as moldable tissue engineering scaffolds," Adv. Mater. 20, 236-239 (2008).

[3] This, H., "Molecular gastronomy," Nat. Mater. 4, 5-7 (2005).

[4] Mewis, J., and N. J. Wagner, "Thixotropy," Adv. Colloid Interface Sci. 147-148, 214-227 (2009).

[5] Lionberger, R. A., and W. B. Russel, "High frequency modulus of hard sphere colloids," J. Rheol. 38, 1885-1908 (1994).

[6] Varga, Z., and J. W. Swan, "Linear viscoelasticity of attractive colloidal dispersions," J. Rheol. 59, 1271-1298 (2015).

[7] Shih, W., Y. Shih, S. Kim, J. Liu, and I. A. Aksay, "Scaling behavior of the elastic properties of colloidal gels," Phys. Rev. A 42, 4772-4780 (1990).

[8] Rueb, C. J., and C. F. Zukoski, "Viscoelastic properties of colloidal gels," J. Rheol. 41, 197-218 (1997).

[9] Buscall, R., P. D. A. Mills, J. W. Goodwin, and D. W. Lawson, "Scaling behaviour of the rheology of aggregate networks formed from colloidal particles," J. Chem. Soc. Faraday Trans. $1 \mathbf{8 4}$, 4249-4260 (1988).

[10] Zaccone, A., H. Wu, and E. Del Gado, "Elasticity of arrested shortranged attractive colloids: Homogeneous and heterogeneous glasses," Phys. Rev. Lett. 103, 208301 (2009).

[11] Bergenholtz, J., and M. Fuchs, "Nonergodicity transitions in colloidal suspensions with attractive interactions," Phys. Rev. E 59, 5706-5715 (1999).

[12] Ramakrishnan, S., Y. L. Chen, K. S. Schweizer, and C. F. Zukoski, "Elasticity and clustering in concentrated depletion gels," Phys. Rev. E 70, 040401 (2004).

[13] Potanin, A. A., R. De Rooij, D. van den Ende, and J. Mellema, "Microrheological modeling of weakly aggregated dispersions," J. Chem. Phys. 102, 5845-5853 (1995).

[14] Hsiao, L. C., R. S. Newman, S. C. Glotzer, and M. J. Solomon, "Role of isostaticity and load-bearing microstructure in the elasticity of yielded colloidal gels," PNAS 109, 16029-16034 (2012).

[15] Hsiao, L. C., H. Kang, K. H. Ahn, and M. J. Solomon, "Role of shearinduced dynamical heterogeneity in the nonlinear rheology of colloidal gels," Soft Matter 10, 9254-9259 (2014).

[16] Larson, R. G., "Constitutive equations for thixotropic fluids," J. Rheol. 59, 595-611 (2015).
[17] Kim, J. M., A. P. R. Eberle, A. Kate Gurnon, L. Porcar, and N. J. Wagner, "The microstructure and rheology of a model, thixotropic nanoparticle gel under steady shear and large amplitude oscillatory shear (LAOS)," J. Rheol. 58, 1301-1328 (2014).

[18] Vermant, J., L. Walker, P. Moldenaers, and J. Mewis, "Orthogonal versus parallel superposition measurements," J. Non-Newtonian Fluid Mech. 79, 173-189 (1998).

[19] Mobuchon, C., P. J. Carreau, and M.-C. Heuzey, "Structural analysis of non-aqueous layered silicate suspensions subjected to shear flow," J. Rheol. 53, 517-538 (2009).

[20] Vermant, J., and M. J. Solomon, "Flow-induced structure in colloidal suspensions," J. Phys. Condens. Matter 17, 187-216 (2005).

[21] Masschaele, K., J. Fransaer, and J. Vermant, "Flow-induced structure in colloidal gels: Direct visualization of model 2D suspensions," Soft Matter 7, 7717-7726 (2011).

[22] Masschaele, K., J. Fransaer, and J. Vermant, "Direct visualization of yielding in model two-dimensional colloidal gels subjected to shear flow," J. Rheol. 53, 1437-1460 (2009).

[23] Rajaram, B., and A. Mohraz, "Microstructural response of dilute colloidal gels to nonlinear shear deformation," Soft Matter 6, 2246-2259 (2010).

[24] Pignon, F., A. Magnin, and J.-M. Piau, "Butterfly light scattering pattern and rheology of a sheared thixotropic clay gel," Phys. Rev. Lett. 79, 4689-4692 (1997).

[25] Varadan, P., and M. J. Solomon, "Shear-induced microstructural evolution of thermoreversible colloidal gel," Langmuir 17, 2918-2929 (2001).

[26] Hoekstra, H., J. Mewis, T. Narayanan, and J. Vermant, "Multi length scale analysis of the microstructure in sticky sphere dispersions during shear flow," Langmuir 21, 11017-11025 (2005).

[27] Hoekstra, H., J. Vermant, J. Mewis, and G. G. Fuller, "Flow-induced anisotropy and reversible aggregation in two-dimensional suspensions," Langmuir 19, 9134-9141 (2003).

[28] Kogan, M., and M. J. Solomon, "Electric-field-induced yielding of colloidal gels in microfluidic capillaries," Langmuir 26, 1207-1213 (2010).

[29] Gurnon, A. K., C. R. Lopez-Barron, A. P. R. Eberle, L. Porcar, and N. J. Wagner, "Spatiotemporal stress and structure evolution in dynamically sheared polymer-like micellar solutions," Soft Matter 10, 2889-2898 (2014).

[30] Frattini, P. L., and G. G. Fuller, "The dynamics of dilute colloidal suspensions subject to time-dependent flow-fields by conservative dichroism," J. Colloid Interface Sci. 100, 506-518 (1984).

[31] Abbasi, S., P. J. Carreau, and A. Derdouri, "Flow induced orientation of multiwalled carbon nanotubes in polycarbonate nanocomposites: Rheology, conductivity and mechanical properties," Polymer 51, 922-935 (2010).

[32] Vermant, J., P. Van Puyvelde, P. Moldenaers, J. Mewis, and G. G. Fuller, "Anisotropy and orientation of the microstructure in viscous emulsions during shear flow," Langmuir 14, 1612-1617 (1998).

[33] Janeschitz-Kriegl, H., Polymer Melt Rheology and Flow-Birefringence (Springer-Verlag, Berlin Heidelberg, 1983).

[34] Osuji, C. O., and D. A. Weitz, "Highly anisotropic vorticity aligned structures in a shear thickening attractive colloidal system," Soft Matter 4, 1388-1392 (2008).

[35] Stauffer, D., Introduction to Percolation Theory (Taylor and Francis, London, 1985).

[36] Brader, J. M., M. E. Cates, and M. Fuchs, "First-principles constitutive equation for suspension rheology," Phys. Rev. E 101, 138301 (2008).

[37] Dullaert, K., and J. J. Mewis, “A model system for thixotropy studies," Rheol. Acta 45, 23-32 (2005).

[38] Vermant, J., P. Moldenaers, J. Mewis, M. Ellis, and R. Garritano, "Orthogonal superposition measurements using a rheometer equipped 
with a force rebalanced transducer," Rev. Sci. Instrum. 68, 4090-4096 (1997).

[39] Yamamoto, M., "Rate-dependent relaxation spectra and their determination,” J. Rheol. 15, 331-344 (1971).

[40] Tanner, R., and J. Simmons, "Combined simple and sinusoidal shearing in elastic liquids,” Chem. Eng. Sci. 22, 1803-1815 (1967).

[41] Kim, S., J. Mewis, C. Clasen, and J. Vermant, "Superposition rheometry of a wormlike micellar fluid," Rheol. Acta 52, 727-740 (2013).

[42] Lin, N. Y. C., X. Cheng, and I. Cohen, "Biaxial shear of confined colloidal hard spheres: The structure and rheology of the vorticity-aligned string phase," Soft Matter 10, 1969-1976 (2014).

[43] Fritz, G., B. J. Maranzano, N. J. Wagner, and N. Willenbacher, "High frequency rheology of hard sphere colloidal dispersions measured with a torsional resonator," J. Non-Newtonian Fluid Mech. 102, 149-156 (2002).

[44] Jacob, A. R., A. S. Poulos, S. Kim, J. Vermant, and G. Petekidis, "Convective cage release in model colloidal glasses," Phys. Rev. Lett. 115, 218301 (2015).

[45] Dullaert, K., and J. Mewis, "Stress jumps on weakly flocculated dispersions: Steady state and transient results," J. Colloid Interface Sci. 287, 542-551 (2005).

[46] Dullaert, K., and J. Mewis, "A structural kinetics model for thixotropy," J. Non-Newtonian Fluid Mech. 139, 21-30 (2006).

[47] Simmons, J. M., "A servo-controlled rheometer for measurement of the dynamic modulus of viscoelastic liquids," J. Sci. Instrum. 43, 887-892 (1966)

[48] Mewis, J., and G. Schoukens, "Mechanical spectroscopy of colloidal dispersions," Faraday Discuss. Chem. Soc. 65, 58-64 (1978).

[49] Eberle, A. P. R., N. Martys, L. Porcar, S. R. Kline, W. L. George, J. M. Kim, P. D. Butler, and N. J. Wagner, "Shear viscosity and structural scalings in model adhesive hard-sphere gels," Phys. Rev. E 89, 050302 (2014).

[50] Martys, N. S., M. Khalil, W. L. George, D. Lootens, and P. Hébraud, "Stress propagation in a concentrated colloidal suspension under shear,” Eur. Phys. J. E 35, 20 (2012).
[51] Martin, J. E., D. W. Schaefer, and A. J. Hurd, "Fractal geometry of vapor-phase aggregates," Phys. Rev. A 33, 3540-3543 (1986).

[52] Vermant, J., G. Cioccolo, K. Golapan Nair, and P. Moldenaers, "Coalescence suppression in model immiscible polymer blends by nano-sized colloidal particles," Rheol. Acta 43, 529-538 (2004).

[53] Willenbacher, N., "Unusual thixotropic properties of aqueous dispersions of Laponite RD,” J. Colloid Interface Sci. 182, 501-510 (1996).

[54] Pignon, F., A. Magnin, and J.-M. Piau, "Thixotropic behavior of clay dispersions: Combinations of scattering and rheometric techniques," J. Rheol. 42, 1349-1373 (1998).

[55] Potanin, A., "Thixotropy and rheopexy of aggregated dispersions with wetting polymer,” J. Rheol. 48, 1279-1293 (2004).

[56] Koumakis, N., E. Moghimi, R. Besseling, W. C. K. Poon, J. F. Brady, and G. Petekidis, "Tuning colloidal gels by shear," Soft Matter 11, 4640-4648 (2015).

[57] Moghimi, E., A. R. Jacob, N. Koumakis, and G. Petekidis, "Colloidal gels tuned by oscillatory shear," Soft Matter 13, 2371-2383 (2017).

[58] Ulrich, G. D., and J. W. Riehl, "Aggregation and growth of submicron oxide particles in flames," J. Colloid Interface Sci. 87, 257-265 (1982).

[59] Walker, L. M., J. Vermant, P. Moldenaers, and J. Mewis, “Orthogonal and parallel superposition measurements on lyotropic liquid crystalline polymers," Rheol. Acta 39, 26-37 (2000).

[60] Sierou, A., and J. F. Brady, "Rheology and microstructure in concentrated noncolloidal suspensions," J. Rheol. 46, 1031-1056 (2002).

[61] Kemp, K. A., and S. V. Letcher, "Ultrasonic determination of anisotropic shear and bulk viscosities in nematic liquid crystals," Phys. Rev. Lett. 27, 1634-1636 (1971).

[62] Cheng, X., X. Xu, S. A. Rice, A. R. Dinner, and I. Cohen, "Assembly of vorticity-aligned hard-sphere colloidal strings in a simple shear flow," PNAS 109, 63-67 (2012).

[63] Xu, X., S. A. Rice, and A. R. Dinner, "Relation between ordering and shear thinning in colloidal suspensions," PNAS 110, 3771-3776 (2013). 